\title{
EVALUATION OF ANTIDIABETIC ACTIVTY OF DIFFERENT EXTRACTS OF MYRISTICA FRAGRANS HOUTT: IN VITRO AND IN SILICO STUDIES
}

\author{
SARANYA SIVARAJ ${ }^{1}$, GOMATHI KANNAYIRAM ${ }^{1}$, GAYATHRI DASARARAJU ${ }^{2 *}$
}

${ }^{1}$ Department of Biotechnology, Dr. M.G.R. Educational and Research Institute University, Chennai - 600 095 , Tamil Nadu, India. ${ }^{2}$ Centre of Advanced Study in Crystallography and Biophysics, University of Madras, Guindy Campus, Chennai - 600 025 , Tamil Nadu, India. Email: drdgayathri@gmail.com

Received: 21 December 2016, Revised and Accepted: 31 December 2016

\section{ABSTRACT}

Objective: This study is aimed to evaluate the antidiabetic effect of sequentially extracted (hexane, dichloromethane [DCM], ethyl acetate, and ethanol) Myristica fragrans houtt (mace) through in vitro and in silico studies.

Methods: The in vitro antidiabetic effect of the sequentially extracted plant was evaluated for its alpha-amylase inhibitory activity, and the potential binding was studied by in silico studies using Schrödinger Maestro.

Results: All extracts showed dose-dependent alpha-amylase inhibitory effect. At concentration $500 \mu \mathrm{g} / \mathrm{ml}$, all the extracts showed more than $60 \%$ inhibition of the alpha-amylase enzyme and the highest inhibition (81.3\%) at $500 \mu \mathrm{g} / \mathrm{ml}$ was observed in DCM extract of mace. Potential compounds were identified by in silico molecular docking studies of alpha-amylase with phytocomponents from DCM extract. Among the top three compounds from virtual screening, induced fit docking studies revealed 2,5-bis(3,4-dimethoxyphenyl)-3, and 4-dimethyloxolane possessed better binding affinity when compared with the drug metformin.

Conclusion: The obtained in vitro and in silico results suggest that all extracts of $M$. fragrans may be used successfully for the management of diabetes mellitus.

Keywords: Myristica fragrans, Mace, Sequential extraction, Alpha-amylase, Molecular docking.

(C) 2017 The Authors. Published by Innovare Academic Sciences Pvt Ltd. This is an open access article under the CC BY license (http://creativecommons. org/licenses/by/4. 0/) DOI: http://dx.doi.org/10.22159/ajpcr.2017.v10i4.16720

\section{INTRODUCTION}

Diabetes mellitus is one of the major concerns worldwide with more than 100 million affected people. It is a chronic condition characterized by hyperglycemia with disturbances of carbohydrate, lipid and protein metabolism, which results in insulin deficiency, insulin action or both. Irrespective of the type of diabetes (I or II), patients are required to control their blood glucose with insulin supplements, oral hypoglycemic agents, exercises, and diet [1]. The incidence of diabetes mellitus is markedly increased worldwide due to modern sedentary lifestyle with consumption of junk foods. Carbohydrates are the major constituents of human diet and play an important role in energy supply. Enzymes like alpha-amylase help in breakdown of dietary carbohydrates to glucose, which can be easily absorbed from intestinal lumen and transported into blood circulation [2]. Hence, inhibition of the enzyme alpha-amylase delays glucose absorption rate and maintains the blood glucose level in hyperglycemic patients. There are various alpha-amylase inhibitors available in the market such as acarbose, miglitol, and voglibose, which produce serious side effects like bloating and abdominal discomfort [3]. The major drawback of these drugs is their non-specificity in targeting different glucosidases. Due to prominent side effects of commercially available drugs have driven seeking for alternative therapies with less severe or no side effects. There are more than 400 traditional plants that are reported to have an antidiabetic effect, but only a few of them are investigated scientifically.

Many plants have been used as a medicine for various deceased conditions since ancient times in traditional medicinal systems such as Chinese, Indian Ayurveda, and Arabic Unani. Plants produce secondary metabolites that possess therapeutic properties used in the treatment of innumerable diseases. Secondary metabolites include tannins, saponins, essential oils, flavonoids, phenols, and alkaloids. Many plant extracts have been reported for their medicinal properties against a variety of diseases such as reactive oxygen species, diabetes mellitus, inflammation, neurodegenerative diseases, antimicrobial [4], and cancer. Spices and herbs have been used in the folk medicine and are the part of the kitchen necessities. Mace (Myristica fragrans) is an aril covering of the seed nutmeg, which is widely used as a flavoring agent in food. The essential oil of the mace contains various phytochemicals such as alpha-pinene, sabinene, alpha-phellandrene, alpha-terpinene, p-cymene, 1,8-cineole, limonene, beta-phellandrene, gamma-terpinen, linalool, safrole, myristicin, and elemicin [5]. Mace is also a part of traditional medicine used to treat indigestion, stimulating the appetite, nausea, to subside aches. Recent studies on mace revealed that the spice also has anti-inflammatory and hepato-protective properties.

In this regard, this study aims to evaluate the antidiabetic potential of four sequentially extracted mace through in vitro and in silico analysis.

\section{METHODS}

\section{Chemicals and reagents}

Alpha-amylase, soluble starch, 3,5-dinitrosalicylic acid (DNS), sodium hydroxide pellets, and sodium potassium tartrate were purchased from Hi-Media Laboratories Pvt. Ltd.

\section{Plant materials and extraction}

The aril part of the M. fragrans (mace) was obtained from Indian spices market and authenticated by Dr. N. Radhakrishnan, Assistant Professor, Centre of Advanced Study in Botany, University of Madras, Guindy Campus, Chennai. The dried mace was powdered and successively extracted with different organic solvents (hexane, dichloromethane (DCM), ethyl acetate, and ethanol) in the increasing polarity order [6]. Initially, $100 \mathrm{~g}$ of powdered mace was soaked in $300 \mathrm{ml}$ of $100 \%$ hexane and incubated for 3 days with intermittent shaking. The extract was filtered using Whatman filter paper No. 1 and dried in a China dish covered with perforated aluminum foil. To the sediments, DCM solvent was added, soaked and filtered; the similar procedure was followed for 
ethyl acetate and ethanol fractions. The extracts were stored at $4^{\circ} \mathrm{C}$ until use. The yield percentage of the extracts was 1.6, 1.4, 2.2, and 1.6 for hexane, DCM, ethyl acetate, and ethanol, respectively.

\section{Alpha-amylase inhibition assay}

Alpha-amylase inhibition assay was performed based on the protocol with minor modifications [7,8]. Different concentrations of plants extracts (50-500 $\mu \mathrm{g} / \mathrm{ml})$ were used to prepare the reaction mixture. The plant extracts were incubated with $0.2 \mathrm{ml}$ of enzyme diluted in $200 \mathrm{mM}$ sodium phosphate buffer at $\mathrm{pH} 6.9$ for 10 minutes at room temperature followed by addition of $0.2 \mathrm{ml}$ of $1 \%$ starch solution and incubated for 15 minutes at room temperature. The reaction was terminated by the addition of $0.5 \mathrm{ml}$ of DNS ( $1 \mathrm{~g}$ of DNS dissolved in $20 \mathrm{ml}$ of 2 $\mathrm{M} \mathrm{NaOH}$, to which $30 \mathrm{~g}$ of sodium potassium tartrate was added and made up to $100 \mathrm{ml}$ with distilled water). Further, the reaction mixture was incubated in boiling water for 15 minutes followed by immediate cooling on ice. Absorbance was measured using ultraviolet visible spectrophotometer (Shimadzu 1650pc) at $540 \mathrm{~nm}$. Positive control was performed with drug acarbose. All the analysis was performed in triplicates. Percentage inhibition by the plant extracts was calculated according to the formula:

Inhibition $(\%)=\operatorname{Abs}_{540}($ control $)-\operatorname{Abs}_{540}($ sample $) \times 100 / \mathrm{Abs}_{540}$ (control)

\section{Molecular docking analysis}

In silico molecular docking studies were conducted with the help of Schrödinger Maestro [9] to identify the binding affinity of the mace compounds toward the enzymes. Three-dimensional structure of the target protein human pancreatic alpha-amylase (protein data bank [PDB] id: 2QV4) [10] was downloaded from PDB. Pytocomponents from DCM extracts were identified by gas chromatography and mass spectrometry and the two-dimensional structures were retrieved from PubChem [11] and minimized using the ligprep module in Schrödinger maestro software. The minimized compounds were then docked at the active site region of the minimized target protein using extra precision (XP) screening followed by an induced fit docking (IFD). The drug metformin was subjected to docking at the active site of the enzyme for comparison studies

\section{Statistical analysis}

Data were analyzed statistically with Statistica/Macsoftware (Prism, USA). The experimental results were mean \pm standard deviation of three parallel measurements. Mean differences were analyzed statistically by running one-way analysis of variance test. $p<0.05$ was considered statistically significant when compared to relevant controls.

\section{RESULTS}

\section{In vitro alpha-amylase inhibitory activity}

The alpha-amylase inhibitory activity of the hexane, ethyl acetate, DCM, and ethanol extracts of mace was shown in the Fig. 1. All the four extracts and the positive control acarbose exhibited a dose-dependent alpha-amylase inhibition activity. At $500 \mu \mathrm{g} / \mathrm{ml}$, DCM extract exhibited alpha-amylase inhibitory potential of $81.3 \%$ which was comparable with the inhibitory potential of acrabose (98.15\%). Among four extracts, DCM and ethanol extracts showed $50 \%$ inhibition of the enzyme at $200 \mu \mathrm{g} / \mathrm{ml}$ whereas, hexane and ethyl acetate showed $40 \%$ inhibition.

\section{Molecular docking studies with human pancreatic alpha-amylase} enzyme

To support the in vitro alpha-amylase inhibition studies and to reveal the binding mechanism of phytocomponents at the active site of alphaamylase, we have performed in silico molecular docking study.

Based on the results of XP virtual screening, top three compounds (2,5-bis(3,4-dimethoxyphenyl)-3,4-dimethyloxolane, ( 2Z) - 2 - hydroxyi min o-N-(4-methoxyphenyl) a ce ta mide, and $\mathrm{N}$-pentafluoropropionyl-3,4-dimethoxyphenylethylamine) were identified and subjected to IFD. Docking score, energy, active site hydrogen bonds, and hydrophobic interactions of compounds were compared with the docking results of metformin at the active site of human pancreatic alpha-amylase (Table 1).

Among the three compounds that were subjected to IFD, 2,5-bis(3,4-dimethoxyphenyl)-3,4-dimethyloxolane showed much better binding affinity on comparing with metformin based on the active site interactions, docking score, and energy. 2,5-bis(3,4dimethoxyphenyl)-3,4-dimethyloxolane strongly binds at the active site of human pancreatic alpha-amylase through the hydrogen bonds with Gln63, Asp197 and hydrophobic interactions with Trp58, Trp59, Tyr62, His101, Gly104, Val107, Leu162, Thr163, Gly164, Leu165, Arg195, Ala198, Glu233, Asp300, His305 amino acid residues (Fig. 2). Docking score, energy for metformin, and 2,5-bis(3,4-dimethoxyphenyl)3,4-dimethyloxolane were found to be $-6.20,-28.16 \mathrm{kcal} / \mathrm{mol}$ and $-6.523,-44.507 \mathrm{kcal} / \mathrm{mol}$, respectively.

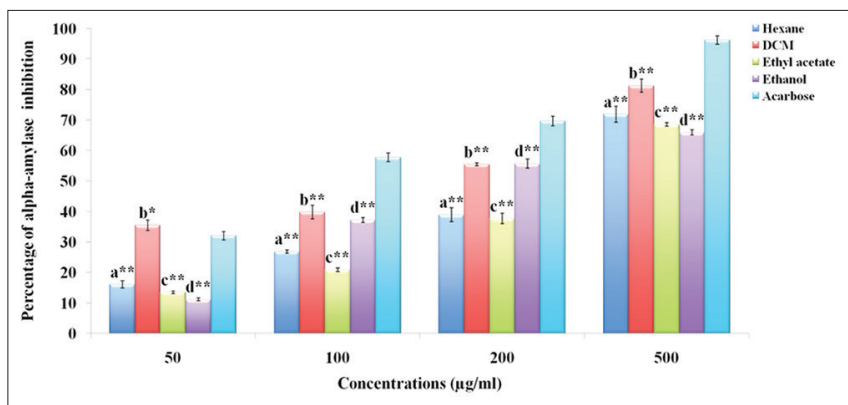

Fig. 1: Inhibitory effects of four extracts of mace against alphaamylase enzyme. Values are expressed in mean \pm standard deviation $(n=3)$, statistical significant test for comparison was done by analysis of variance followed by Dunnett's t-test comparison between (a) Hexane versus acarbose, (b) dichloromethane versus acarbose, (c) ethyl acetate versus acarbose, and (d) ethanol versus acarbose. ${ }^{*} \mathbf{p}<0.05$, ${ }^{* *} \mathbf{p}<0.1$ and NS: Nonsignificant

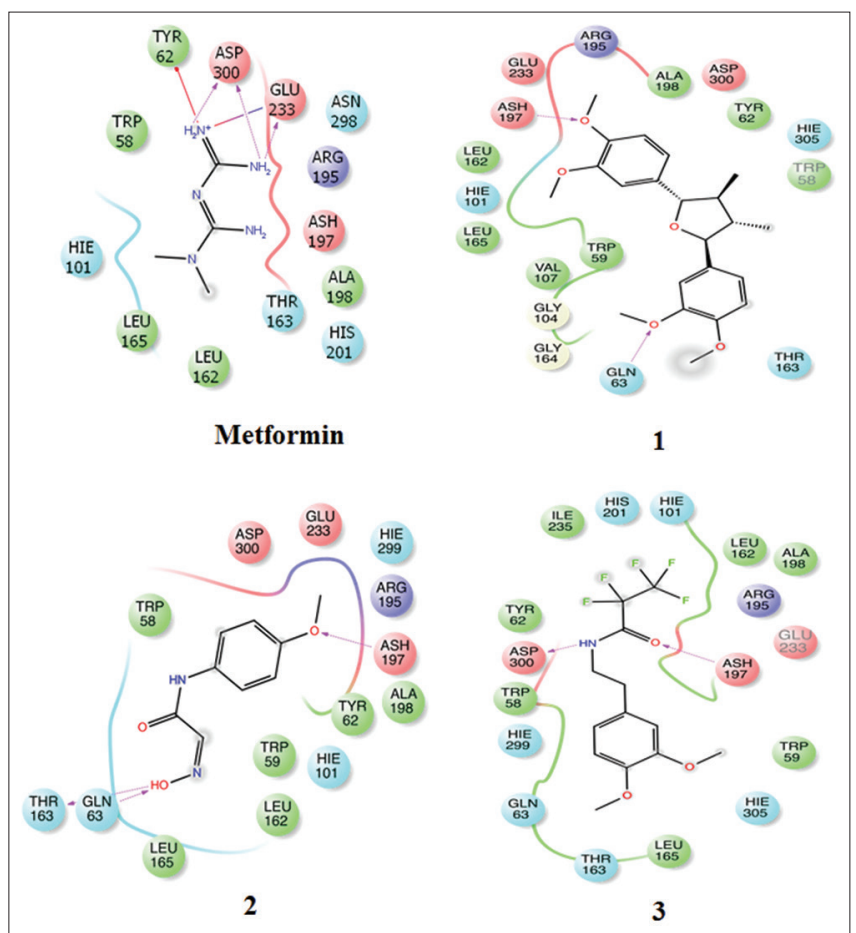

Fig. 2: Hydrogen bonds and hydrophobic interactions at the active site of human pancreatic alpha-amylase enzyme. 2,5-bis(3,4-dimethoxyphenyl)-3,4-dimethyloxolane (1), (2Z)-2-hydroxyimino-N-(4-methoxyphenyl)acetamide (2), N-pentafluoropropionyl-3,4-dimethoxyphenylethylamine (3) 
Table 1: IFD results of DCM extracts of $M$. fragrans houtt

\begin{tabular}{lll}
\hline Compound & Docking score \\
\hline Metformin & -6.20 \\
2,5-bis (3,4-dimethoxyphenyl)-3,4-dimethyloxolane & -6.523 & -28.16 \\
(2Z)-2-hydroxyimino-N-(4-methoxyphenyl) acetamide & -6.204 & -44.507 \\
N-pentafluoropropionyl-3,4-dimethoxyphenylethylamine & -5.788 & -33.022 \\
\hline
\end{tabular}

IFD: Induced fit docking, DCM: Dichloromethane, M. fragrans: Myristica fragrans

\section{DISCUSSION}

As diabetes is a multifactorial disease leading to several complications, it demands a multiple therapeutic approaches. Although several therapies are in use, there are certain limitations such as high cost and side effects such as development of hypoglycemia, weight gain, gastrointestinal disturbances, and liver toxicity [12]. The pharmacotherapy of diabetes has recently undergone unprecedented expansion. However, the challenge is to optimize glycemic control with a minimum number of medication while taking into consideration the cost of the therapy, adverse effect profiles, ease of administration, and the urgency for blood sugar normalization. The search for new pharmacologically active agents obtained by screening natural sources such as medicinal plants or their extracts has led to the discovery of many clinically useful drugs that play a major role in the treatment of diabetes. A number of medicinal plants and their formulations are used for treating diabetes in folklore/Ayurvedic medicine system as well as in ethno medicinal practices.

This study clearly demonstrated the antidiabetic potential of the sequentially extracted mace through its in vitro and in silico alphaamylase inhibition studies. Most of the previous studies were done on seed part of the plant called nutmeg, which acts as a hypoglycemic agent by reducing the blood sugar level significantly. Our findings reveal that all the four extracts of mace inhibited the alpha-amylase enzyme in concentration dependent manner particularly DCM extract possessed the maximum inhibitory activity which can be comparable with acarbose. Plants are rich sources of therapeutic agents and are known to treat various human ailments over millennia. It is generally accepted that a cumulative synergistic relationship among the phytochemicals is responsible for the overall beneficial medicinal value of plants. The previous reports suggest that phenolic [13] and flavonols [14] are the compounds that modulate the enzymatic breakdown of carbohydrate by inhibiting alpha-amylase enzyme. Since maximum alpha-amylase enzyme inhibition was shown by the DCM extract, in silico molecular docking studies were performed for the phytocomponents present in the DCM extract alone. The alpha-amylase inhibitory effect of various phytocomponents of DCM extracts was revealed through in silico studies and the results suggested the high binding potential of 2,5-bis(3,4dimethoxyphenyl)-3,4-dimethyloxolane at the active site of alphaamylase enzyme when compared with other phytocomponents and with the control drug metformin. Natural sources and semi-synthetic derivatives of natural products play a crucial role in development of novel drugs in the modern pharmaceutical industries $[15,16]$. Hence, the compound 2,5-bis(3,4-dimethoxyphenyl)-3,4-dimethyloxolane present in the DCM extract can be further screened for its antidiabetic activity, which may serve as a potential drug candidate like acarbose or metformin in controlling diabetes and its associated complications.

\section{CONCLUSION}

The obtained results suggest that all the four extracts can form a good source of effective inhibitors of alpha-amylase enzyme and may be used as a potential drug in the treatment of diabetes mellitus. Further investigations are required for commercial usage.

\section{REFERENCES}

1. Rahimzadeh M, Jahanshahi S, Moein S, Moein MR. Evaluation of alpha-amylase inhibition by Urtica dioica and Juglans regia extracts. Iran J Basic Med Sci 2014;17(6):465-9.

2. Keerthana G, Kalaivani MK, Sumathy A. In-vitro alpha-amylase inhibitory and antioxidant activities of ethanolic leaf extract of Corton bonplandianum. Asian J Pharm Clin Res 2013;6(4):32-6.

3. Bhutkar MA, Bhise SB. In vitro assay of alpha amylase inhibitory activity of some indigenous plants. Int J Chem Sci 2012;10(1):457-62.

4. Perumal Samy R, Gopalakrishnakone P. Therapeutic potential of plants as anti-microbials for drug discovery. Evid Based Complement Alternat Med 2010;7(3):283-94.

5. Nagja T, Vimal K, Sanjeev A. Myristica fragrans: A comprehensive review. Int J Pharm Pharm Sci 2016;8(2):27-30.

6. Jeyaseelan EC, Jenothiny S, Pathmanathan MK, Jeyadevan JP. Antibacterial activity of sequentially extracted organic solvent extracts of fruits, flowers and leaves of Lawsonia inermis L. From Jaffna. Asian Pac J Trop Biomed 2012;2(10):798-802.

7. Sangeetha N, Rintu B. Optimization of culture parameters to enhance production of amylase and protease from Aspergillus awamori in a single fermentation. Afr J Bio Res 2010;4(3):73-80.

8. Kalpana S, Ramakrushna B, Anitha S. Evaluation of in vitro antioxidant and $\alpha$-amylase inhibitory activity of Phyllanthus indofischeri bennet. Int J Pharm Pharm Sci 2016;8(11):131-6.

9. Farid R, Day T, Friesner RA, Pearlstein RA. New insights about HERG blockade obtained from protein modeling, potential energy mapping, and docking studies. Bioorg \& Med Chem 2006;14:3160-73.

10. Maurus R, Begum A, Williams LK, Fredriksen JR, Zhang R, Withers SG, et al. Alternative catalytic anions differentially modulate human alphaamylase activity and specificity. Biochemistry 2008;47(11):3332-44.

11. Kim S, Thiessen PA, Bolton EE, Chen J, Fu G, Gindulyte A, et al. PubChem substance and compound databases. Nucleic Acids Res 2016;44(D1):D1202-13

12. Dey L, Attele AS, Yuan CS. Alternative therapies for Type 2 diabetes. Altern Med Rev 2002;7(1):45-58.

13. McDougall GJ, Shpiro F, Dobson P, Smith P, Blake A, Stewart D. Different polyphenolic components of soft fruits inhibit alpha-amylase and alpha-glucosidase. J Agric Food Chem 2005;53(7):2760-6.

14. Bhanudas NM. Evaluation of alpha amylase inhibitory potential of four traditional culinary leaves. Asian J Pharm Clin Res 2012;5(2):75-6.

15. Cragg GM, Newman DJ. Natural products: A continuing source of novel drug leads. Biochim Biophys Acta 2013;1830(6):3670-95.

16. Dharmendra KK, Archana RJ. $\alpha$-glucosidase and $\alpha$-amylase inhibitory activity of Indigofera cordifolia seeds and leaves extract. Int J Pharm Pharm Sci 2014;6(11):152-5. 